\title{
Notas sobre esta edición
}

\author{
Mario Zúñiga
}

Director, Cuadernos de Antropología

Universidad de Costa Rica, San José, Costa Rica

zn.mario@gmail.com

Para esta edición de Cuadernos de Antropología, presentamos una serie de documentos, los cuales tienen que ver con temáticas propias de la Arqueología, la Antropología Social y la memoria de nuestra disciplina.

El primer documento en nuestro número es un reconocimiento a la trayectoria y aportes del antropólogo y lingüista, Dr. Otto Schumman Gálvez, quien murió en marzo de 2015, y dejó un gran vacío entre quienes estudian la lengua y cultura mesoamericanas. La antropóloga lingüista y profesora de nuestra escuela, Dra. Giselle Chang, ha redactado este sentido homenaje, desde un punto de vista personal, y rescata los aportes del Dr. Schumman a la academia costarricense como especialista y como un "maestro del diálogo" de trato amable y sencillo.

El segundo texto es un extenso artículo escrito por el Dr. David F. Mora-Marín de la Universidad de Carolina del Norte y se titula "The 'Charlie Chaplin' Silhouette Figural Theme: A Pan-Middle American Ritual Performer Theme". En él se exploran las representaciones de la figura conocida como "Charles Chaplin" en el jade costarricense y se contrastan con las que se producen en otras partes de Mesoamérica.

Cuadernos de Antropología

Enero-Junio 2016, 26(1), 5-7

Revista del Laboratorio de Etnología María Eugenia Bozzoli Vargas

Escuela de Antropología, Universidad de Costa Rica

http://revistas.ucr.ac.cr/index.php/antropologia

ISSN 2215-356X 
Con base en esto, el autor postula la interesante tesis de que esta imagen tiene continuidad no solo en la región mesoamericana, sino más hacia el Sur. Se trabaja sobre el concepto de Axis Mundi, mediante el cual se postula el carácter regional de la representación y su continuidad.

Por su parte, la Dra. Leila Rodríguez Soto, del Departamento de Antropología de la Universidad de Cincinatti, publica un artículo titulado "Valorizando los efectos de las emigraciones y las remesas: una comunidad costarricense ante la (re)estructuración de jerarquías de género y clase". En este, se trabajan los resultados de una investigación realizada en una comunidad del sur de Costa Rica con un importante flujo migratorio. El artículo trabaja sobre el concepto de "economía moral" y analiza, desde el punto de vista de quienes no migran, las transformaciones de la vida social y cultural desarrollada en una comunidad debido al fenómeno de la migración.

El número cuenta además con los textos de dos noveles y prometedores antropólogos. El primero, el artículo del Diego Montero Hernández, "Una interpretación del conflicto por el desabastecimiento de agua potable ocurrido durante el 2014 en el cantón de Barva de Heredia, Costa Rica"; toma como base la información de su tesis de licenciatura en Antropología Social. En él se explora la problemática del desabastecimiento de agua y las respuestas políticas que da la comunidad por medio de la conformación del Foro Cantonal Barveño en Defensa del Agua, cuyo impacto es visible tanto en las actividades públicas como en el ciberespacio, a través de las redes sociales.

La sección de artículos se cierra con el segundo, el trabajo de Mario Araya-Perez, “Es la cárcel violenta en sí misma? Violencia y legitimación de la dinámica institucional en el Centro de Formación Juvenil Zurquí, Heredia, Costa Rica". Este trabajo también se desprende de una tesis de licenciatura en Antropología Social y aborda el siempre difícil concepto de "violencia" para comprenderlo en la dinámica de un centro penal. Este texto parte de una reflexión que sitúa la discusión sobre el concepto y que, posteriormente, se complementa con los datos surgidos en los diarios de campo elaborados por el autor en su investigación.

Asimismo, en esta edición, nos complacemos en publicar también una separata escrita por la Dra. Maria Eugenia Bozzoli Vargas, como material base para la lección inaugural que se dio en la Escuela de Antropología de la Universidad de Costa Rica en mayo de 2015. El texto se titula "Algunos hitos en el devenir de la disciplina antropológica en Costa Rica: 1880-2015" y otorga una perspectiva pormenorizada y completa del devenir de la disciplina en el país. Es un texto que, de seguro, se convertirá en un valioso material de referencia para comprender la historia de la disciplina antropológica en particular, además de la historia de las ciencias sociales en Costa Rica. 
Por último, aparece en esta publicación, un comentario de la Lic. Ana Cecilia Arias Quirós a propósito del libro de reciente publicación “La mirada antropológica de María Eugenia Bozzoli, 1960-1985” (2015) escrito y compilado por Olga Echeverría Murray y Margarita Bolaños Arquín en la Editorial de la Universidad Estatal a Distancia. Este comentario fue leído por su autora en la muestra efectuada en la Escuela de Antropología, el 15 de junio de 2016. La otra presentadora del texto fue la Dra. Eugenia Ibarra, cuyo texto será publicado en el Anuario de Estudios Centroamericanos con el propósito de dar a la obra la mayor difusión posible. 